\title{
Anesthetic Implications of Malarial Infection in a Child
}

\author{
Shabana Z. Shafy a, d, e, Mohammed Hakim a, d, Renee Henga, d, \\ Joseph D. Tobias ${ }^{a, b, c, d}$
}

\begin{abstract}
Malaria is a life-threatening mosquito-borne parasitic disease, with a wide clinical spectrum and multisystem involvement. It is associated with high rates of mortality and morbidity worldwide, particularly in Africa and Southeast Asia. Cases are now being reported in developed countries resulting from global and international travel. Given the varied presentation and multisystem involvement, patients with malaria may present for surgery, thereby necessitating anesthetic care. We present a 4-year-old child from Burkina Faso, Africa, with a known diagnosis of Plasmodium falciparum malaria, who presented for anesthetic care for a magnetic resonance imaging of the lumbar spine, requiring general anesthesia. The etiology and presentation of malaria are discussed and its end-organ involvement is reviewed with emphasis on the anesthetic implications of the disease.
\end{abstract}

Keywords: Malaria; General anesthesia; Plasmodium falciparum

\section{Introduction}

Malaria is a life-threatening multi-organ systemic infectious disease that is endemic throughout most of the tropics. In 2015, malaria was reported to be endemic in 91 countries and territories in the tropics and subtropics, with approximately half of the world population at risk for infection. The World Health Organization (WHO) estimated that 212 million cases of malaria occurred worldwide in 2015, resulting in 429,000 deaths. The African region accounts for an estimated $92 \%$ of all malaria deaths with Plasmodium falciparum and vivax causing the majority of the morbidity and mortality worldwide [1-3].

The Plasmodium parasite is transmitted by the infected fe-

Manuscript submitted February 19, 2019, accepted March 4, 2019

aDepartment of Anesthesiology \& Pain Medicine, Nationwide Children's Hospital, Columbus, OH, USA

${ }^{b}$ Department of Pediatrics, The Ohio State University College of Medicine, Columbus, OH, USA

'Department of Anesthesiology \& Pain Medicine, The Ohio State University College of Medicine, Columbus, OH, USA

${ }^{\mathrm{d}}$ These authors contributed equally to this manuscript.

${ }^{\text {e}}$ Corresponding Author: Shabana Zainab Shafy, Department of Anesthesiology \& Pain Medicine, Nationwide Children's Hospital, 700 Children's Drive, Columbus, OH 43205, USA. Email: shabana.shafy@nationwidechildrens.org

doi: https://doi.org/10.14740/jmc3267 male Anopheles mosquito. The bite from an infected mosquito introduces Plasmodium sporozoites from the mosquito's saliva into the patient's blood. Following introduction into the bloodstream, the sporozoites travel to the liver and undergo maturation. The Plasmodium life cycle consists of exoerythrocytic and erythrocytic stages. During the exoerythrocytic phase, the sporozoites invade hepatocytes and divide until schizonts are formed containing thousands of merozoites. The schizonts rupture and release merozoites into the bloodstream, which invade red blood cells (erythrocytic phase). Asexual reproduction within erythrocytes eventually results in hemolysis of the red blood cells, releasing thousands more merozoites into the bloodstream. This release can result in a pro-inflammatory cytokines response. The released merozoites may then infect new red cells, while others differentiate into gametocytes, which circulate in the bloodstream until they are ingested by a mosquito to continue the transmission cycle [2].

Malaria is a multisystem disease with a varied clinical spectrum based on the parasite species, epidemiology, immunity, and age. Groups at highest risk include young children and pregnant women. Features of complicated malaria may include cerebral malaria, renal impairment, non-cardiogenic pulmonary edema, anemia, thrombocytopenia, and hepatic dysfunction. Patients with malaria may present for anesthetic care during radiological imaging or surgical procedures $[4,5]$. We present a case of a 4-year-old child, with $P$. falciparum malaria infection, secondary anemia and thrombocytopenia, who required anesthetic care for magnetic resonance imaging (MRI) of lumbar spine. The multisystem involvement of the disease process is presented and anesthetic implications are discussed.

\section{Case Report}

Institutional Review Board approval is not required for presentation of single case reports at Nationwide Children's Hospital (Columbus, OH). At the time of surgery, the patient was a 4-year-old, 15.4-kg child, from Burkina Faso, Africa, who was scheduled for MRI of the lumbar spine under general anesthesia. Her past history was significant for an anorectal malformation, which had previously required surgical correction with a colostomy. She was also diagnosed with $P$. falciparum infection 1 week prior to this hospitalization after presenting with intermittent episodes of high-grade fever. She had subsequently developed thrombocytopenia and anemia secondary to the malarial infection. There was no history of previous problems with general anesthesia during her surgical procedures. Her immunization status was incomplete and she had no known 
allergies. Current medications included atovaquone/proguanil $\mathrm{HCl}$ (Malarone), acetaminophen and ibuprofen oral suspension, and multivitamins with iron oral drops. On the morning of the procedure, her physical examination and preoperative vital signs were unremarkable. Preoperative laboratory studies demonstrated hemoglobin of $8.9 \mathrm{~g} / \mathrm{dL}$, hematocrit $28.6 \%$, and a normal platelet count. Renal and hepatic function tests had normalized. After the initiation of treatment, the peripheral blood smear demonstrated no parasites. The anesthetic plan, risks, benefits and alternatives to general anesthesia were discussed with the parent and informed consent for general anesthesia was obtained. The patient was held nil per os for 6 $\mathrm{h}$ and was transported to the operating room. After arrival in the operating room (OR), the patient was placed in the supine position and standard American Society of Anesthesiologists (ASA) monitors were placed. The induction of anesthesia was carried out with the incremental inhalation of sevoflurane in nitrous oxide and oxygen. After anesthetic induction, peripheral intravenous access was obtained under aseptic conditions with a 22-gauge intravenous catheter, and a laryngeal mask airway (LMA) was placed. The patient was then positioned, padded and secured under the MRI scanner for imaging of the lumbar spine. Maintenance anesthesia included sevoflurane in air/oxygen with spontaneous ventilation. Additional medications included dexamethasone $(4 \mathrm{mg})$, ondansetron $(1.5 \mathrm{mg})$, glycopyrrolate $(0.15 \mathrm{mg})$, and dexmedetomidine $(5 \mu \mathrm{g})$. Intraoperative fluids included $250 \mathrm{~mL}$ of lactated Ringers. The MRI was completed with no intraoperative complications. The total anesthetic time was $60 \mathrm{~min}$. At the completion of the procedure, the LMA was removed and, the patient was transported to the post-anesthesia care unit (PACU) in stable condition. She was discharged to home later that day and her post-discharge course was unremarkable.

\section{Discussion}

Malaria should be suspected as a potential diagnosis in patients with a febrile illness if they lived in or visited an endemic region. The initial symptoms of uncomplicated malaria are nonspecific and can vary greatly, with non-specific presenting features including tachycardia, tachypnea, fever with chills, fatigue, diaphoresis, headache, anorexia, nausea, vomiting, abdominal pain, diarrhea, and myalgia [3]. Regardless of the species involved, a malaria infection may result in no clinical symptoms and be considered uncomplicated. However, such patients may still transmit the disease.

$P$. falciparum is most commonly associated with complicated malaria, although patients with severe vivax infection may also manifest severe end-organ involvement including pulmonary complications and adult respiratory distress syndrome (ARDS). Major risk factors for increasing severity of infection include immunocompromised and asplenic patients, children 6 - 59 months of age, and pregnant women. Some of the common manifestations of severe malaria which can herald a grave prognosis and warrant immediate treatment include altered consciousness with or without seizures, circulatory collapse, ARDS, hepatic and renal failure, acidosis and severe hy- poglycemia. Cerebral malaria is the most fatal complication of malaria. It is an encephalopathy presenting with altered consciousness with or without seizures and neurologic deficits, arising from multifactorial causes including, sequestration of malarial parasitized erythrocytes within cerebral capillaries, impairment of the blood-brain barrier, parasite-induced apoptosis, and tissue degeneration, as well as decline in neuroprotective angiogenic growth factors [3, 6-8].

Changes in the structure and function of parasitized erythrocytes are implicated in the pathogenesis of severe falciparum malaria. The deformability of the erythrocyte cell membrane is decreased and its osmotic fragility is increased because of the parasites within the erythrocytes. Specific membrane glycoproteins are subsequently expressed leading to adherence of erythrocytes to the surface of the vascular endothelium (cytoadherence), resulting in microvascular obstruction of capillaries and small venules. The parasitized erythrocytes also trigger the release of pro-inflammatory cytokines, leading to systemic inflammatory effects, fever, hyperdynamic circulation, and direct myocardial suppression. These effects may result in mortality from severe malaria through impairment of both mitochondrial function and oxygen utilization [9-11]. Additionally, it is imperative to rule out concomitant infections. There is a proven association between malaria, human immunodeficiency virus (HIV), malnutrition, and invasive bacterial infections. The immunosuppression caused by HIV increases the severity and degree of parasitemia, hindering the treatment process [12].

Microscopic visualization of the parasite within erythrocytes in Giemsa-stained films is standardly used to diagnose malaria, although a single negative blood film does not rule out the diagnosis. Visible parasitemia on the blood film may be obscured by sequestration of the parasites within deep tissues. Other investigations used as an adjunct for diagnosis include rapid diagnostic tests for specific malarial antigens or enzymes. However, these do not quantify the level of parasitemia which is crucial in monitoring the response to treatment $[13,14]$. Laboratory evaluations may also demonstrate non-specific findings such as anemia, thrombocytopenia, elevated transaminases, mild coagulopathy, and elevated blood urea nitrogen (BUN) and creatinine.

The treatment strategies include those aimed at eradicating the disease causing organism and those aimed and preventing remission of disease. Prompt assessment and initiation of antimalarial therapy are essential, followed by concurrent supportive care to manage the life-threatening end-organ complications of the disease. The WHO has laid out treatment guidelines based on species subtype and disease severity, with the first-line treatment options being primarily focused on the use of artemisinin-based combination therapies, chloroquine and primaquine [4]. Additional supportive care aimed at treating severe anemia with transfusion of blood, intravenous fluids, appropriate management of coagulopathy, and measures towards replenishment of nutritional stores should be implemented.

Patients with malarial infections may present for surgery. As with any anesthetic care, the first step is a thorough preoperative examination with identification of associated co-morbid conditions as well as end-organ involvement of the disease. 
One of the most common presenting conditions requiring surgery for patients with malaria is tropical splenomegaly with splenic rupture due to traumatic or non-traumatic causes. Plasmodium vivax and ovale remain dormant in the liver for long durations, and the stress associated with anesthesia and surgery may induce relapse [15-17]. Complete general and system-wise physical examination, along with preoperative laboratory investigations including a complete blood count, blood type with cross-match, electrolytes, coagulation profile, renal and hepatics function tests, blood glucose and lactate, chest $\mathrm{X}$-ray and electrocardiography should be performed. General intraoperative anesthetic goals adopted in patients with malaria include maintaining hemodynamic stability, avoiding cerebral vasodilation and alterations of intracranial pressure (ICP), lung protective ventilation strategies with attention to peak inflating pressure (PIP) and the application of positive end expiratory pressure (PEEP) as needed, avoidance of fluid overload and hypoglycemia, and the transfusion of blood and blood products [4].

\section{Cardiac system}

In severe malaria, ischemic cardiomyopathy due to microvascular occlusion of coronary arteries has been reported on postmortem examination. Conduction defects and electrocardiogram abnormalities may occur in patients on quinine therapy. Elevated levels of $\mathrm{N}$-terminal pro-brain natriuretic peptide (BNP), indicating left ventricular dysfunction, has been demonstrated in some patients [18-20].

\section{Respiratory system}

Respiratory involvement with cough and ARDS may occur with severe falciparum malaria. Respiratory compromise can arise from the effects of severe anemia, associated pneumonia, non-cardiogenic pulmonary edema, cardiac dysfunction, and metabolic acidosis. Preoperative assessment of respiratory function with continuous intraoperative attention to respiratory mechanics including PIP along with adoption of lung protective strategies is suggested. In those with concurrent cerebral malaria, protective measures aimed at maintaining normocarbia and use of extracorporeal carbon dioxide removal devices may be beneficial [21]

\section{Central nervous system (CNS)}

Obstruction of the cerebral microvasculature by parasitized erythrocytes has been implicated in the pathologic process leading to cerebral symptoms. This can lead to hypoxia, ischemia, increased ICP, and seizures. Cerebral malaria is the most common fatal neurological presentation of falciparum malaria and is diagnosed by confirmation of parasitemia coupled with altered consciousness, in the absence of other causes of coma. Volatile anesthetic agents should be used with caution as they may cause cerebral vasodilatation and increase ICP
$[22,23]$. Severe CNS involvement may necessitate continuous ICP monitoring and aggressive therapies to treat elevated ICP. Benzodiazepines (lorazepam or midazolam), barbiturates, propofol or other standard anticonvulsant agents can be used to treat seizures.

\section{Hematologic system}

The hematologic complications include anemia, thrombocytopenia and coagulopathy. Thrombocytopenia and anemia are secondary to immune-mediated lysis and splenic sequestration of platelets and parasitized erythrocytes. Activation of the coagulation cascade and fibrinolytic pathway, with release of tissue factor, are implicated in the pathogenesis of coagulation disturbances and disseminated intravascular coagulation which may occur with severe malaria [24].

\section{Gastrointestinal system}

Hepatic dysfunction, if present in $P$. falciparum, indicates a poor prognosis. The spleen is the main organ involved in the destruction and removal of parasitized erythrocytes. Impairment of splenic function can impair the immune response to malarial parasites and antigens. The hyper-reactive malarial splenomegaly syndrome (HMS) is a leading cause of massive splenomegaly in malaria-endemic countries and is caused by chronic antigenic stimulation by malarial parasite. The enlarged spleen can be injured in blunt abdominal trauma with splenic rupture presenting as hemoperitoneum or hemothorax. Acute acalculous cholecystitis has been reported in children with falciparum and vivax malaria $[16,25,26]$.

\section{Metabolic system}

Malaria is often complicated by hypoglycemia, the symptoms of which may be masked by general anesthesia. Hypoglycemia results from diminished hepatic glycogen stores, hyperpyrexia, and impaired hepatic gluconeogenesis with anaerobic glycolysis. Hypoglycemia may also be an adverse effect of quinine therapy. Metabolic acidosis may result from impaired hepatic function, tissue hypoxia and renal impairment. Parasite lactate production, anaerobic glycolysis, tissue hypoxia and secondary to microvascular obstruction are the main factors for development of lactic acidosis. Fever is a common clinical finding, which under general anesthesia, along with the hyper-metabolic state seen in malaria, may lead to an increased end-tidal carbon dioxide level, being mistaken for malignant hyperthermia [27-29]. Therefore, careful observation and perioperative monitoring of temperature and vital signs are essential in malarial patients. Patients with normal coagulation function including platelet counts may be candidates for regional anesthetic techniques as an alternative to general anesthesia. However, use of neuraxial anesthesia in patients at risk for secondary bacterial infections with bacteremia is not without risk. The development of meningitis and vertebral col- 
Table 1. Previous Reports of Anesthetic Care in Malaria

\begin{tabular}{|c|c|c|}
\hline Authors and reference & Cohort demographics & Summary of anesthetic care and outcome \\
\hline $\begin{array}{l}\text { Fareed and } \\
\text { Mahmoud [17] }\end{array}$ & $\begin{array}{l}\text { A } 29 \text {-year-old male patient presenting } \\
\text { with hepato-splenomegaly and } \\
\text { splenic rupture with hemoperitoneum } \\
\text { requiring emergency laparotomy. }\end{array}$ & $\begin{array}{l}\text { Emergency laparotomy for splenectomy was successively performed after } \\
\text { four units of whole blood transfusion. Histopathology of the specimen } \\
\text { showed malarial pigments in macrophages and congested red pulp, } \\
\text { confirming diagnosis of a malarial spleen. Antimalarial drugs were } \\
\text { administered postoperatively. }\end{array}$ \\
\hline Mathew et al [31] & $\begin{array}{l}\text { A } 26 \text {-year-old woman, presenting at } \\
28 \text { weeks gestation for emergency } \\
\text { cesarean section for severe sepsis. A } \\
\text { preliminary diagnosis of malaria was } \\
\text { made and confirmed postoperatively. }\end{array}$ & $\begin{array}{l}\text { RSI with thiopental and succinylcholine. Maintenance anesthesia with } \\
\text { isoflurane in 50\% nitrous oxide. PEEP of } 5 \mathrm{~cm} \mathrm{H}_{2} \mathrm{O} \text { was required to } \\
\text { maintain oxygenation. A 1,140-g infant was delivered and required } \\
\text { ventilator support. Intraoperatively, empiric antibiotics were administered } \\
\text { along with the transfusion of two units of red blood cells. Postoperatively, } \\
\text { the diagnosis of malaria was confirmed. }\end{array}$ \\
\hline Zanfini et al [32] & $\begin{array}{l}\text { A } 27 \text {-year-old woman, with known } \\
P . \text { falciparum infection, presenting } \\
\text { at } 28 \text { weeks of gestation. }\end{array}$ & $\begin{array}{l}\text { Emergent cesarean section under spinal anesthesia with bupivacaine } \\
\text { and sufentanil. A } 1.77-\mathrm{kg} \text { infant was delivered with respiratory distress, } \\
\text { requiring oxygen support. The postoperative course of the mother was } \\
\text { uneventful. }\end{array}$ \\
\hline Yagmur et al [34] & $\begin{array}{l}\text { Two patients ( } 56 \text { and } 32 \text { years } \\
\text { of age) with splenic rupture, } \\
\text { both being treated for malaria } \\
\text { at the time of presentation. }\end{array}$ & $\begin{array}{l}\text { Exploratory laparotomy with splenectomy performed in both cases with } \\
\text { free blood in the peritoneal cavity and an enlarged ruptured spleen. The } \\
\text { postoperative courses were uneventful. }\end{array}$ \\
\hline
\end{tabular}

RSI: rapid sequence induction; PEEP: positive end expiratory pressure; ICU: intensive care unit.

umn abscess after regional anesthesia is rare, with sepsis and pregnancy being major risk factors. Recommended guidelines by the American Society of Regional Anesthesia (ASRA) are that patients with evidence of systemic infection may safely undergo spinal anesthesia after appropriate antibiotic therapy has been initiated and a response to therapy has been seen [30].

To date, there are only a limited number of reports in the literature regarding anesthetic care for patients with malaria (Table 1) [17, 31-34]. Anesthesia providers should be mindful of the end-organ effects of the disease process. A relevant history of the presenting illness, a thorough physical examination, and careful preoperative assessment of diagnostic laboratory investigations to identify the pathogen and assess the severity of disease are key to effective perioperative care. Intraoperative monitoring and choice of anesthetic agents are chosen based on the end-organ effects of the disease and the anticipated physiologic impact of the specific anesthetic agents.

\section{Acknowledgments}

Not applicable.

\section{Funding}

None.

\section{Conflict of Interest}

None.

\section{References}

1. Ashley EA, Pyae Phyo A, Woodrow CJ. Malaria. Lancet. 2018;391(10130):1608-1621.

2. Daily JP, Scanfeld D, Pochet N, Le Roch K, Plouffe D, Kamal M, Sarr O, et al. Distinct physiological states of Plasmodium falciparum in malaria-infected patients. Nature. 2007;450(7172):1091-1095.

3. White NJ. The treatment of malaria. N Engl J Med. 1996;335(11):800-806.

4. Soltanifar D, Carvalho B, Sultan P. Perioperative considerations of the patient with malaria. Can J Anaesth. 2015;62(3):304-318.

5. SaaChai T, Lin J. Anesthetic aspect of malaria disease: a brief review. Middle East J Anaesthesiol. 2012;21(4):457462.

6. McGready R, Wongsaen K, Chu CS, Tun NW, Chotivanich $\mathrm{K}$, White NJ, Nosten F. Uncomplicated Plasmodium vivax malaria in pregnancy associated with mortality from acute respiratory distress syndrome. Malar J. 2014;13:191.

7. Steketee RW, Nahlen BL, Parise ME, Menendez C. The 
burden of malaria in pregnancy in malaria-endemic areas. Am J Trop Med Hyg. 2001;64(1-2 Suppl):28-35.

8. Armah HB, Wilson NO, Sarfo BY, Powell MD, Bond VC, Anderson W, Adjei AA, et al. Cerebrospinal fluid and serum biomarkers of cerebral malaria mortality in Ghanaian children. Malar J. 2007;6:147.

9. David PH, Hommel M, Miller LH, Udeinya IJ, Oligino LD. Parasite sequestration in Plasmodium falciparum malaria: spleen and antibody modulation of cytoadherence of infected erythrocytes. Proc Natl Acad Sci U S A. 1983;80(16):5075-5079.

10. Clark IA, Budd AC, Alleva LM, Cowden WB. Human malarial disease: a consequence of inflammatory cytokine release. Malar J. 2006;5:85.

11. Prato M, Giribaldi G, Polimeni M, Gallo V, Arese P. Phagocytosis of hemozoin enhances matrix metalloproteinase- 9 activity and TNF-alpha production in human monocytes: role of matrix metalloproteinases in the pathogenesis of falciparum malaria. J Immunol. 2005;175(10):6436-6442.

12. Berkley JA, Bejon P, Mwangi T, Gwer S, Maitland K, Williams TN, Mohammed S, et al. HIV infection, malnutrition, and invasive bacterial infection among children with severe malaria. Clin Infect Dis. 2009;49(3):336-343.

13. Trampuz A, Jereb M, Muzlovic I, Prabhu RM. Clinical review: Severe malaria. Crit Care. 2003;7(4):315-323.

14. Bell D, Peeling RW, Pacific/TDR WH-ROftW. Evaluation of rapid diagnostic tests: malaria. Nat Rev Microbiol. 2006;4(9 Suppl):S34-38.

15. Gibney EJ. Surgical aspects of malaria. Br J Surg. 1990;77(9):964-967.

16. Gupta N, Lal P, Vindal A, Hadke NS, Khurana N. Spontaneous rupture of malarial spleen presenting as hemoperitoneum: a case report. J Vector Borne Dis. 2010;47(2):119120.

17. Fareed MI, Mahmoud AE. Spontaneous rupture of falciparum malarial spleen presenting as hemoperitoneum, hemothorax, and hemoarthrosis. Am J Case Rep. 2013;14:405-408.

18. Mohsen AH, Green ST, West JN, McKendrick MW. Myocarditis associated with Plasmodium falciparum malaria: a case report and a review of the literature. J Travel Med. 2001;8(4):219-220.

19. Padmaja UK, Adhikari P, Periera P. Experience with quinine in falciparum malaria. Indian J Med Sci. 1999;53(4):153-157.

20. Ehrhardt S, Wichmann D, Hemmer CJ, Burchard GD, Brattig NW. Circulating concentrations of cardiac proteins in complicated and uncomplicated Plasmodium falciparum malaria. Trop Med Int Health. 2004;9(10):1099-1103.

21. Cove ME, MacLaren G, Federspiel WJ, Kellum JA. Bench to bedside review: Extracorporeal carbon dioxide removal, past present and future. Crit Care. 2012;16(5):232.

22. Idro R, Ndiritu M, Ogutu B, Mithwani S, Maitland K, Berkley J, Crawley J, et al. Burden, features, and outcome of neurological involvement in acute falciparum malaria in Kenyan children. JAMA. 2007;297(20):2232-2240.

23. Idro R, Marsh K, John CC, Newton CR. Cerebral malaria: mechanisms of brain injury and strategies for improved neurocognitive outcome. Pediatr Res. 2010;68(4):267274.

24. Francischetti IM. Does activation of the blood coagulation cascade have a role in malaria pathogenesis? Trends Parasitol. 2008;24(6):258-263.

25. Leoni S, Buonfrate D, Angheben A, Gobbi F, Bisoffi Z. The hyper-reactive malarial splenomegaly: a systematic review of the literature. Malar J. 2015;14:185.

26. Poddighe D, Tresoldi M, Licari A, Marseglia GL. Acalculous acute cholecystitis in previously healthy children: general overview and analysis of pediatric infectious cases. Int J Hepatol. 2015;2015:459608.

27. White NJ, Warrell DA, Chanthavanich P, Looareesuwan S, Warrell MJ, Krishna S, Williamson DH, et al. Severe hypoglycemia and hyperinsulinemia in falciparum malaria. N Engl J Med. 1983;309(2):61-66.

28. Day NP, Phu NH, Mai NT, Chau TT, Loc PP, Chuong LV, Sinh DX, et al. The pathophysiologic and prognostic significance of acidosis in severe adult malaria. Crit Care Med. 2000;28(6):1833-1840.

29. Delas Alas V, Geddes LA, Voorhees WD, Bourland JD, Schoenlein WE. End-tidal $\mathrm{CO} 2, \mathrm{CO} 2$ production, and $\mathrm{O} 2$ consumption as early indicators of approaching hyperthermia. Biomed Instrum Technol. 1990;24(6):440-444.

30. Wedel DJ, Horlocker TT. Regional anesthesia in the febrile or infected patient. Reg Anesth Pain Med. 2006;31(4):324-333.

31. Mathew DC, Loveridge R, Solomon AW. Anaesthetic management of caesarean delivery in a parturient with malaria. Int J Obstet Anesth. 2011;20(4):341-344.

32. Zanfini BA, Dell'Anna AM, Catarci S, Frassanito L, Vagnoni S, Draisci G. Anesthetic management of urgent cesarean delivery in a parturient with acute malaria infection: a case report. Korean J Anesthesiol. 2016;69(2):193-196.

33. Pandey RK, Batra MM, Darlong V, Garg R, Punj J, Kumar S. Anesthetic management of parturient with thoracic kyphoscoliosis, malaria and acute respiratory distress syndrome for urgent cesarean section. J Anaesthesiol Clin Pharmacol. 2015;31(4):558-559.

34. Yagmur Y, Kara IH, Aldemir M, Buyukbayram H, Tacyildiz IH, Keles C. Spontaneous rupture of malarial spleen: two case reports and review of literature. Crit Care. 2000;4(5):309-313. 